\title{
Generalizing the Shift Method for Rectangular Shaped Vertices with Visibility Constraints ${ }^{\star}$
}

\author{
Seok-Hee Hong ${ }^{1}$ and Martin Mader ${ }^{2}$ \\ ${ }^{1}$ School of IT, University of Sydney, NSW, Australia \\ ${ }^{2}$ Department of Computer and Information Science, University of Konstanz, Germany
}

\begin{abstract}
In this paper we present a generalization of the shift method algorithm [4]6] to obtain a straight-line grid drawing of a triconnected graph, where vertex representations have a certain specified size. We propose vertex representations having a rectangular shape. Additionally, one may demand maintainance of the criterion of strong visibility, that is, any possible line segment connecting two adjacent vertices cannot cross another vertex' representation. We prove that the proposed method produces a straight-line grid drawing of a graph in linear time with an area bound, that is only extended by the size of the rectangles, compared to the bound of the original algorithm.
\end{abstract}

\section{Introduction}

The shift method [4] is a well-known method among several approaches to obtain a standard straight-line representation of planar graphs in the graph drawing literature [2/7/9]. Given a triangulated graph, the original algorithm calculates coordinates for each vertex on an 2D integer grid such that the final drawing has a quadratic area bound. A linear time variant is presented in [3], [6] provides a version for triconnected graphs, [5] for biconnected graphs.

The approach presented in the following sections is related to a version of the shift method given in [1], which allows square vertex representations. In this paper, the shift method for triconnected graphs [6] is generalized to have rectangular shaped vertex representations. Furthermore, we demand that the criterion of strong visibility between adjacent vertices is satisfied, that is, any possible line segment connecting two adjacent vertices does not cross another vertex' representation. To maintain the strong visibility criterion in the shift method, additional shifts have to be introduced. The main contribution is to prove that the proposed method produces a grid drawing with an area quadratic in the sum of number of vertices and the sizes of the vertex representations.

The generalized shift method can be used to draw clustered graphs having planar quotient graphs [8]. Other possible applications include drawing graphs that have arbitrary vertex representations by using the minimal bounding box, or drawing graphs with labeled vertices, where the positions of a vertex and its label are not known, but only the size of the region into which they are allowed to be drawn.

\footnotetext{
* This work was supported by DFG Research Training Group GK-1042 "Explorative Analysis and Visualization of Large Information Spaces”, University of Konstanz.
} 

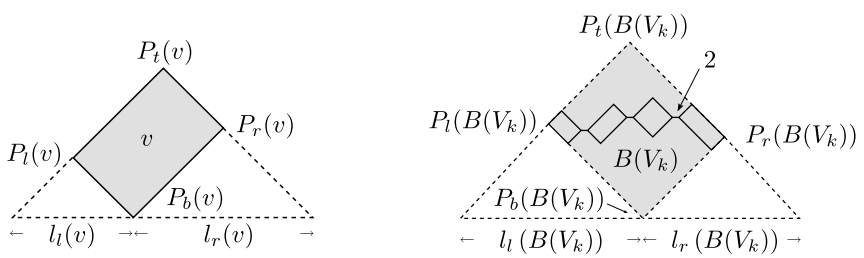

Fig. 1. Vertex representations. Left: singleton $V_{k}=\{v\}$. Right: $\left|V_{k}\right|>1$.

\section{Preliminaries}

Let $G=(V, E)$ be a graph with $n=|V|$ and $m=|E|$. A graph is called planar if it has a crossing-free drawing in the plane. A plane graph is a planar graph with a fixed cyclic ordering of edges incident to each vertex and a fixed outer face. A plane graph divides the plane into which it is drawn into connected regions called faces. A triconnected graph is a graph where the removal of any pair of vertices does not disconnect the graph.

Let $G$ be a triconnected plane graph. Let $\pi=\left(V_{1}, V_{2}, \ldots, V_{K}\right), K<n$, be a lmcordering of $G$ as presented in [6]. It is shown that every triconnected plane graph has a $l m c$-ordering, and it can be computed in linear time. Let $G_{k}, k \leq K$, be the graph induced by $V_{1} \cup \cdots \cup V_{k}$ according to $\pi$, particularly $G_{K}=G$. We denote by $C_{0}\left(G_{k}\right)$ the boundary of the outer face of $G_{k}$.

Vertices are represented as rectangles rotated by 45 degrees. For all $v \in V$, vertex lengths $l_{l}(v)$ and $l_{r}(v)$ are given according to the side lengths of a vertex representation, as illustrated in Fig 1] Let $l(v)=l_{l}(v)+l_{r}(v)$. Let $P_{l}(v), P_{r}(v), P_{b}(v)$ and $P_{t}(v)$ be the left, right, bottom and top corners of $v$ 's representation, with $P_{l}(v)=\left(x_{l}(v), y_{l}(v)\right)$, etc. As illustrated in Fig 1, we represent a set $V_{k}=\left\{v_{k}^{1}, \ldots, v_{k}^{j}\right\}, j>1$, as a chain of the single vertices, where $\left[P_{r}\left(v_{k}^{i}\right), P_{l}\left(v_{k}^{i+1}\right)\right], 1 \leq i<j$, are horizontally aligned with distance two. Let $l\left(V_{k}\right)=\sum_{v \in V_{k}} l(v), l_{l}\left(V_{k}\right)=\sum_{v \in V_{k}} l_{l}(v)$, and $l_{r}\left(V_{k}\right)$ accordingly. Let $B\left(V_{k}\right)$ be the minimal bounding box of the representation of $V_{k}$. For a singleton $V_{k}=\left\{v_{k}\right\}$, the corner points of $B\left(V_{k}\right)$ are exactly the corner points of $v_{k}$. To obtain a grid drawing, we assume without loss of generality that $l_{l}(v), l_{r}(v) \in \mathbb{N}_{0}$ for all $v \in V$ and both are even.

For vertex representations having an area, as the representation given above, we can define the criterion of strong visibility for graph drawing algorithms:

Definition 1 (Strong visibility). Let $v, w \in V$. Then $v$ is strongly visible to $w$, if any line segment connecting a point within the representation of $v$ to a point within the representation of $w$ does not cross the representation of any other vertex $u \in V$ with $u \neq v, w$.

Let $P_{1}$ and $P_{2}$ be two grid points on an integer grid and let $\mu\left(P_{1}, P_{2}\right)$ be the intersection point of the straight-line segment with slope +1 through $P_{1}$ and the straight-line segment with slope -1 through $P_{2}$. In the algorithm, vertices will be placed according to $\mu$; hence the rotation of vertex representations by 45 degrees. Let $L(v)$ be a set of 

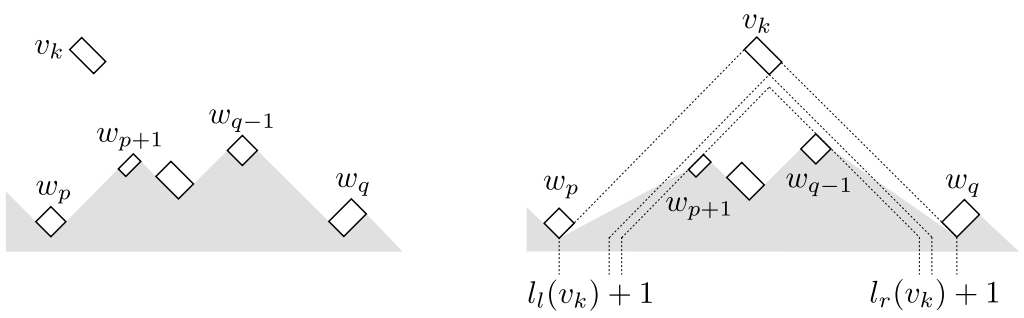

Fig. 2. Installing vertex $v_{k}$. Left: $G_{k-1}$. Right: $G_{k}$.

dependent vertices of $v$, that will later on contain the vertices which have to be rigidly moved with $v$ when $v$ itself is moved.

\section{Algorithm}

The algorithm starts by drawing $G_{2}$. We place $V_{1}=\left\{v_{1}^{1}, v_{1}^{2}\right\}$ and $V_{2}$ with coordinates $P_{r}\left(v_{1}^{1}\right) \leftarrow(0,0), P_{l}\left(v_{1}^{2}\right) \leftarrow\left(l\left(V_{2}\right)+\max \left\{l_{l}\left(v_{1}^{1}\right), l_{r}\left(v_{1}^{2}\right)\right\}+2 \cdot\left|V_{2}\right|, 0\right)$ and $P_{t}\left(B\left(V_{2}\right)\right) \leftarrow$ $\mu\left(P_{r}\left(v_{1}^{1}\right), P_{l}\left(v_{1}^{2}\right)\right)$. The sets of dependent vertices are initialized with $L(v) \leftarrow\{v\}$ for $v \in G_{2}$. We proceed by placing the next set $V_{k}$ in the $l m c$-ordering into $G_{k-1}$, one by one, starting with $V_{3}$. Let $C_{0}\left(G_{k-1}\right)=w_{1}, \ldots, w_{t}, w_{1}=v_{1}$ and $w_{t}=v_{2}$. Assume that following conditions hold for $G_{k-1}, k \geq 3$ :

(C1) $x_{r}\left(w_{i}\right)<x_{l}\left(w_{i+1}\right), 1 \leq i \leq t-1$.

(C2) each straight-line segment $\left(P_{r}\left(w_{i}\right), P_{l}\left(w_{i+1}\right)\right), 1 \leq i \leq t-1$, has either slope $+1,0$ or -1 .

(C3) every vertex in $G_{k-1}$ is strongly visible to its adjacent vertices in $G_{k-1}$.

Obviously, these conditions hold for the initial Graph $G_{2}$. When inserting $V_{k}$, let $w_{1}, \ldots$, $w_{p}, w_{p+1}, \ldots, w_{q}, \ldots, w_{t}$ be the vertices on $C_{0}\left(G_{k-1}\right)$, where $w_{p}$ is the leftmost and $w_{q}$ the rightmost adjacent vertex of $V_{k}$ in $G_{k-1}$. Similar to [36], install $V_{k}=\left\{v_{k}^{1}, \ldots\right.$, $\left.v_{k}^{j}\right\}$ by applying the following steps, see Fig 2 .

Step 1. for all $v \in \bigcup_{i=p+1}^{q-1} L\left(w_{i}\right)$ do $x(v) \leftarrow x(v)+l_{l}\left(V_{k}\right)+\left|V_{k}\right|$

Step 2. for all $v \in \bigcup_{i=q}^{t} L\left(w_{i}\right)$ do $x(v) \leftarrow x(v)+l_{l}\left(V_{k}\right)+l_{r}\left(V_{k}\right)+2 \cdot\left|V_{k}\right|+\Delta$ Step 3. $P_{t}\left(B\left(V_{k}\right)\right) \leftarrow \mu\left(P_{r}\left(w_{p}\right), P_{l}\left(w_{q}\right)\right)$

Step 4. For one $j^{\prime}, 1 \leq j^{\prime} \leq j$ set $L\left(v_{k}^{j^{\prime}}\right) \leftarrow\left\{v_{k}^{j^{\prime}} \cup\left(\bigcup_{i=p+1}^{q-1} L\left(w_{i}\right)\right)\right\}$; for all other $j^{\prime \prime} \neq j^{\prime}, 1 \leq j^{\prime \prime} \leq j$ set $L\left(v_{k}^{j^{\prime \prime}}\right) \leftarrow\left\{v_{k}^{j^{\prime \prime}}\right\}$

Actually, if $V_{k}$ is not a singleton, the bottom corner of $B\left(V_{k}\right)$ is placed too low by $\left|V_{k}\right|-1$. Nevertheless, this is sufficient since every vertex in $V_{k}$ is separated by distance two, and therefore the lowest possible bottom corner of any $v \in V_{k}$ is at least $\left|V_{k}\right|-1$ higher than $P_{b}\left(B\left(V_{k}\right)\right)$. Assume for the moment that $\Delta=0$ in step 2 . Then all conditions are satisfied for $G_{k}$ if $\left\{w_{p+1}, \ldots, w_{q-1}\right\} \neq \emptyset$, see [8]. However, if there are no inner vertices between $w_{p}$ and $w_{q}$ on the outer face of $G_{k-1}$, and $l_{l}\left(w_{p}\right), l_{r}\left(w_{q}\right) \neq 0$, 
condition (C3) is violated in $G_{k}$ by placing $V_{k}$ in steps 1 to 4 , as $w_{q}$ is not strongly visible to $w_{p}$ anymore after insertion. Since step 1 will be omitted in this case, the problem can only be addressed by introducing an extra shift $\Delta$ in step 2, thus placing $V_{k}$ high enough in step 3 such that the strong visibility between $w_{p}$ and $w_{q}$ is not violated in $G_{k}$. The following Lemma shows how much extra shift is needed, when installing $V_{k}$.

Lemma 1. Let $V_{k}=\left\{v_{k}\right\}$. Let $\left\{w_{p+1}, \ldots, w_{q-1}\right\}=\emptyset$ and $l_{l}\left(w_{p}\right), l_{r}\left(w_{q}\right) \neq 0$. Then $w_{p}$ will be strongly visible to $w_{q}$ in $G_{k}$, if an extra shift amount $\Delta$ is added in step 2 with

$$
\Delta=\left\{\begin{array}{cl}
{\left[2 \cdot \frac{l_{l}\left(w_{p}\right) \cdot l_{r}\left(w_{q}\right)}{l_{l}\left(w_{p}\right)+l_{l}\left(w_{q}\right)+l_{r}\left(w_{q}\right)}\right]} & \text { if }\left[P_{r}\left(w_{p}\right), P_{l}\left(w_{q}\right)\right] \text { has slope }+1 \text { in } G_{k-1} \\
{\left[2 \cdot \frac{l_{l}\left(w_{p}\right) \cdot l_{r}\left(w_{q}\right)-4}{l_{l}\left(w_{p}\right)+l_{r}\left(w_{q}\right)+4}\right]} & \text { if }\left[P_{r}\left(w_{p}\right), P_{l}\left(w_{q}\right)\right] \text { has slope } 0 \text { in } G_{k-1} \\
{\left[2 \cdot \frac{l_{l}\left(w_{p}\right) \cdot l_{r}\left(w_{q}\right)}{l_{l}\left(w_{p}\right)+l_{r}\left(w_{p}\right)+l_{r}\left(w_{q}\right)}\right]} & \text { if }\left[P_{r}\left(w_{p}\right), P_{l}\left(w_{q}\right)\right] \text { has slope }-1 \text { in } G_{k-1}
\end{array}\right.
$$

Proof. Let $\delta$ be the height, with which $v_{k}$ must be lifted upwards to guarantee strong visibility. Assume $\left[P_{r}\left(w_{p}\right), P_{l}\left(w_{q}\right)\right]$ has slope +1 in $G_{k-1}$, as illustrated in Fig 3 (left). The gray rectangle indicates the position of $v_{k}$ in $G_{k}$ without introducing an extra shift. Let $\delta_{p q}=\sqrt{2} \cdot \overline{\left[P_{r}\left(w_{p}\right), P_{l}\left(w_{q}\right)\right]}$. Observe that $\delta$ is largest, if $\delta_{p q}$ has the smallest possible value, and that at the same time $\delta_{p q} \geq l_{l}\left(w_{q}\right)$. Thus, assume $\delta_{p q}=l_{l}\left(w_{q}\right)$. By the theorem on intersecting lines, we have

$$
\frac{\delta}{l_{l}\left(w_{p}\right)}=\frac{l_{r}\left(w_{q}\right)}{l_{l}\left(w_{p}\right)+l_{l}\left(w_{q}\right)+l_{r}\left(w_{q}\right)} \quad \Leftrightarrow \quad \delta=\frac{l_{l}\left(w_{p}\right) \cdot\left|w_{q}\right| r}{l_{l}\left(w_{p}\right)+l_{l}\left(w_{q}\right)+l_{r}\left(w_{q}\right)}
$$

It is easy to see that $\delta$ is analogous, if the line segment $\left[P_{r}\left(w_{p}\right), P_{l}\left(w_{q}\right)\right]$ has slope -1 in $G_{k-1}$. Assume $\left[P_{r}\left(w_{p}\right), P_{l}\left(w_{q}\right)\right]$ has slope 0 in $G_{k-1}$, as shown in Fig 3 (right). In this case, $P_{r}\left(w_{p}\right)$ and $P_{l}\left(w_{q}\right)$ are separated by a horizontal line segment with length two. Assume that $l_{l}\left(w_{p}\right)<l_{r}\left(w_{q}\right)$, then

$$
\begin{aligned}
\delta+1 & =\frac{l_{l}\left(w_{p}\right)}{2}+\frac{l_{l}\left(w_{p}\right) / 2+1}{l_{l}\left(w_{p}\right) / 2+2+l_{r}\left(w_{q}\right) / 2} \cdot \frac{l_{r}\left(w_{q}\right)-l_{l}\left(w_{p}\right)}{2} \\
\Leftrightarrow \quad \delta & =\frac{l_{l}\left(w_{p}\right) \cdot l_{r}\left(w_{q}\right)-4}{l_{l}\left(w_{p}\right)+l_{r}\left(w_{q}\right)+4}
\end{aligned}
$$

The same value is obtained, if $l_{l}\left(w_{p}\right) \geq l_{r}\left(w_{q}\right)$. Overall, if an extra shift $\Delta=\lceil 2 \delta\rceil$ is introduced, $v_{k}$ is lifted by at least $\delta$, and hence $w_{p}$ and $w_{q}$ will be strongly visible to each other in $G_{k}$.

Observe that, if $V_{k}$ is not a singleton, we have to add $2 \cdot\left(\left|V_{k}\right|-1\right)$ to $\Delta$, since $P_{b}\left(B\left(V_{k}\right)\right)$ is $\left|V_{k}\right|-1$ lower than the bottom corner of a singleton $v_{k}$, as indicated in Fig 3 . Note also that, if $\Delta$ is an odd number, it has to be increased by one to maintain the grid drawing property.

\section{Analysis}

The following theorems state the bounds for the drawing area of the proposed method, and its time complexity. 

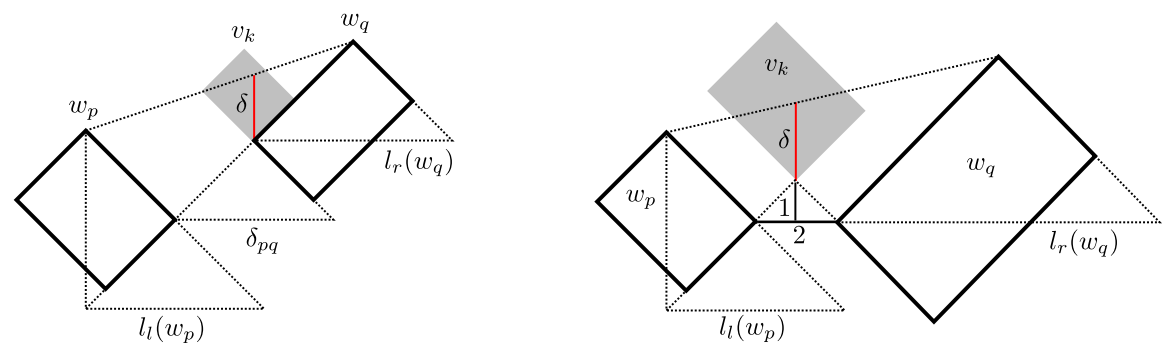

Fig. 3. Geometry for the case $\left\{w_{p+1}, \ldots, w_{q-1}\right\}=\emptyset$. Left: slope +1 . Right: slope 0 .

Theorem 1. The total grid area of a drawing of a triconnected plane graph $G=(V, E)$ with given vertex lengths $l_{l}(v), l_{r}(v), v \in V$ produced by the proposed method is in $O\left(|V|+\sum_{v \in V} l(v)\right)^{2}$.

Proof. The width of the initial layout of $G_{2}$ is clearly bounded by $2 \cdot\left|V_{2}\right|+\Delta_{2}+$ $\sum_{i=1}^{2} l\left(V_{i}\right)$, with $\Delta_{2}=\max \left(l_{l}\left(v_{1}^{1}\right), l_{r}\left(v_{1}^{2}\right)\right)$. Whenever a set $V_{k}$ is added, the width increases by $2 \cdot\left|V_{k}\right|+\Delta_{k}+l\left(V_{k}\right)$, where $\Delta_{k}$ denotes the extra shift in step $k$. Thus, the total width is bounded by $2 \cdot|V|+\sum_{v \in V} l(v)+\sum_{i=2}^{K} \Delta_{i}$.

Assume that all $V_{k}, 2<k \leq K$, are singleton, and that, instead of shifting exactly with $\Delta=\lceil 2 \delta\rceil$ when installing $V_{k}$, we shift with either $\max \left(l_{l}\left(w_{p}\right), l_{r}\left(w_{q}\right)\right)$ or $\min \left(l_{l}\left(w_{p}\right), l_{r}\left(w_{q}\right)\right)$. If $\left[P_{r}\left(w_{p}\right), P_{l}\left(w_{q}\right)\right]$ has slope +1 in $G_{k-1}$ and
1. $\quad l_{l}\left(w_{p}\right) \geq \delta_{p q}+l_{r}\left(w_{q}\right)$
$\delta \leq l_{l}\left(w_{p}\right) / 2$
2. $l_{r}\left(w_{q}\right) \leq l_{l}\left(w_{p}\right)<\delta_{p q}+l_{r}\left(w_{q}\right) \Rightarrow \delta \leq l_{r}\left(w_{q}\right) / 2$,
3. $\quad l_{r}\left(w_{q}\right) \geq l_{l}\left(w_{p}\right)+\delta_{p q}$
$\delta \leq l_{r}\left(w_{q}\right) / 2$,
$\Delta=l_{l}\left(w_{p}\right)$
4. $l_{l}\left(w_{p}\right)<l_{r}\left(w_{q}\right)<l_{l}\left(w_{p}\right)+\delta_{p q}$
$\delta \leq l_{l}\left(w_{p}\right) / 2$,
$\Delta=l_{r}\left(w_{q}\right)$
$\Delta=l_{r}\left(w_{q}\right)$
$\Delta=l_{l}\left(w_{p}\right)$

are sufficient to maintain strong visibility. If $\left[P_{r}\left(w_{p}\right), P_{l}\left(w_{q}\right)\right]$ has slope -1 in $G_{k-1}$, the bounds are analogous. If $\left[P_{r}\left(w_{p}\right), P_{l}\left(w_{q}\right)\right]$ has slope $0, \delta$ is bounded by $\max \left(l_{l}\left(w_{p}\right)\right.$, $\left.l_{r}\left(w_{q}\right)\right) / 2$, therefore we assume to shift with the maximum length in this case. To find an upper bound for $\sum \Delta$ we use amortized analysis.

Consider the part of $\sum \Delta$ which is contributed due to shifting with the maximum length of $l_{l}\left(w_{p}\right)$ and $l_{r}\left(w_{q}\right)$, i.e. cases 1 and 3 , and the case where the slope of $\left[P_{r}\left(w_{p}\right), P_{l}\left(w_{q}\right)\right]$ is 0 . It is easy to see that, after one of these cases occured on one side of a vertex $v$ at step $k$, the length of $v$ on the same side only contributes to another extra shift at step $k^{\prime}>k$ as the minimum length of the two adjacent vertices of $V_{k^{\prime}}$. Hence, this part of $\sum \Delta$ is bounded by $\sum_{v \in V} l(v)$.

For determining the part of $\sum \Delta$ which is contributed due to shifting with the minimum length, let each vertex $v$ have two amounts left $(v)$ and $\operatorname{right}(v)$, that it can spend to support one extra shift on its left side and one on its right side. Set left $(v) \leftarrow l_{r}(v)$ and $\operatorname{right}(v) \leftarrow l_{l}(v)$. Let $w_{p}$ and $w_{q}$ be the neighbors of $V_{k}$ on the outer face of $G_{k-1}$ at step $k$ with $\left\{w_{p+1}, \ldots, w_{q-1}\right\}=\emptyset$. Assume $\left[P_{r}\left(w_{p}\right), P_{l}\left(w_{q}\right)\right]$ has slope +1 in $G_{k-1}$. Since in this case $w_{q}$ was inserted later than $w_{p}$, it cannot have spent left $\left(w_{q}\right)$, because otherwise there would be an inner vertex between $w_{p}$ and $w_{q}$ on the outer face. If $\min \left\{l_{l}\left(w_{p}\right), l_{r}\left(w_{q}\right)\right\}=l_{r}\left(w_{q}\right)$, then $w_{q}$ pays for the extra shift with left $\left(w_{q}\right)$. 
Suppose now that $\min \left\{l_{l}\left(w_{p}\right), l_{r}\left(w_{q}\right)\right\}=l_{l}\left(w_{p}\right)$. If $w_{p}$ has not used $\operatorname{right}\left(w_{p}\right)$ so far, then it just pays for the shift. If on the other hand $\operatorname{right}\left(w_{p}\right)$ has already been spent (e.g. to insert $w_{q}$ ), then $w_{q}$ uses $\operatorname{left}\left(w_{q}\right)=l_{r}\left(w_{q}\right) \geq l_{l}\left(w_{p}\right)$ to pay the extra shift. The payment is analogous if $\left[P_{r}\left(w_{p}\right), P_{l}\left(w_{q}\right)\right]$ has slope -1 in $G_{k-1}$. Thus, the total amount of extra shift is sufficiently paid, and this part of $\sum \Delta$ is therefore also bounded by $\sum_{v \in V} l(v)$. The additional amount of extra shift which is contributed, if $V_{k}$ are not singleton, is clearly bounded by $2 \cdot \sum_{2 \leq i \leq K}\left(\left|V_{k}\right|-1\right)<2 \cdot|V|$.

Since $G=G_{K}$ satisfies condition (c) 2 ), the height of the drawing is bounded by half of its width plus the part of vertices $v_{1}^{1}$ and $v_{1}^{2}$ beneath the X-axis.

If the strong visibility constraint has not to be maintained, the drawing area is exactly $\left(\frac{l\left(v_{1}^{1}\right)+l\left(v_{1}^{2}\right)}{2}+2 \omega\right) \times\left(\frac{\max \left(l_{r}\left(v_{1}^{1}\right), l_{l}\left(v_{1}^{2}\right)\right)}{2}+\omega\right), \omega=|V|-2+\sum_{i=2}^{K} \frac{l\left(V_{i}\right)}{2}$, since no extra shift is needed in this case. It remains an open problem to give a worst-case scenario and sharp area bound if strong visibility has to be guaranteed.

The linear time implementation of the original shift method [3] can easily be extended to our problem. Since the determination of the extra shift amount takes only constant time, the overall asymptotic complexity is not changed.

Theorem 2. Given a triconnected plane graph $G=(V, E), n=|V|$, the proposed method can be implemented with running time $O(n)$.

\section{References}

1. Barequet, G., Goodrich, M.T., Riley, C.: Drawing planar graphs with large vertices and thick edges. J. Graph Algorithms Appl. 8, 3-20 (2004)

2. Battista, G.D., Eades, P., Tamassia, R., Tollis, I.G.: Graph Drawing: Algorithms for the Visualization of Graphs. Prentice-Hall, Englewood Cliffs (1999)

3. Chrobak, M., Payne, T.H.: A linear-time algorithm for drawing a planar graph on a grid. Information Processing Letters 54(4), 241-246 (1995)

4. de Fraysseix, H., Pach, J., Pollack, R.: How to draw a planar graph on a grid. Combinatorica 10(1), 41-51 (1990)

5. Harel, D., Sardas, M.: An algorithm for straight-line drawing of planar graphs. Algorithmica 20(2), 119-135 (1998)

6. Kant, G.: Drawing planar graphs using the canonical ordering. Algorithmica 16(1), 4-32 (1996)

7. Kaufmann, M., Wagner, D. (eds.): Drawing graphs: Methods and Models. Springer, London (2001)

8. Mader, M., Hong, S.: Drawing planar clustered graphs in 2.5 dimensions. Tech. rep., NICTA (2007), http: / /www.cs.usyd.edu.au/ visual/valacon/

9. Nishizeki, T., Rahman, S.: Planar Graph Drawing. Lecture Note Series on Computing, vol. 12. World Scientific, Singapore (2004) 